\title{
EMDR Resource Methods for Women With Breast Cancer
}

\author{
Katy Murray \\ Olympia, WA
}

\begin{abstract}
The diagnosis and treatment of breast cancer can involve a series of life-altering, traumatic experiences, leading to distress, reduced quality of life, and long-standing fears of recurrence. Distress may exacerbate physical symptoms, impair coping, impact health behaviors, and reduce compliance with cancer treatment. Psychological treatments have focused on alleviating distress and improving quality of life. Given the growing evidence of the role of stress and behavior on cancer outcomes, a biobehavioral approach to treatment may serve to achieve these traditional treatment goals while also impacting disease course. Shapiro's (2001) eye movement desensitization and reprocessing (EMDR) protocol for illness and somatic disorders was greatly informed by her interest in mind-body approaches to health and the newly emerging (1992) field of psychoneuroimmunology. The protocol includes first identifying and addressing the realistic fears and challenges a woman faces. Once appropriate, EMDR therapy turns to the reprocessing of past memories, present triggers, and the development of positive future templates of health. This article focuses on situations, usually in the early diagnosis and intensive phases of cancer treatment, when the reprocessing phases of EMDR therapy need to be delayed because of the demands of cancer treatment or insufficient client stability. Resourcing interventions that are compatible with biobehavioral goals are provided, with brief clinical examples.
\end{abstract}

Keywords: breast cancer; cancer-related distress; biobehavioral; EMDR; resourcing

$4 \mathbf{Y}$ ou have breast cancer." These are the words that women dread - words that can be seared into memory by the emotional intensity of the experience (Phelps \& Sharot, 2008). One in 8 women is diagnosed with breast cancer, with $40 \%$ reporting intense fear, helplessness, or horror upon receiving the news (Matsuoka, 2006). Women with breast cancer suffer psychologically from the experiences of uncertainty and loss and physical affects inherent in diagnosis and treatment as well as fears of recurrence and fears of dying (Holland, Herdman, $\&$ Hewitt, 2004). Many experience physical conditions (e.g., fatigue, sleep disturbances, and pain) and psychological distress (e.g., depression, anxiety, fear of recurrence, loneliness, memory and concentration, sexual and body image problems) that can persist over time and adversely affect their overall quality of life (Chopra \& Kamal, 2012).

\section{Breast Cancer and Distress}

During breast cancer diagnosis, treatment, and followup, a woman's perceived life threat is more likely to play a greater role in distress and cancer adjustment than objective disease characteristics (e.g., cancer stage; Laubmeier \& Zakowski, 2004). Perceived lack of social support, coping strategies of hopelessness/ helplessness, anxious preoccupation, cognitive avoidance, and trait dissociation also contribute to distress following cancer diagnosis and treatment (Pérez et al., 2014). Distress can interfere with coping, including the physical symptoms and treatment of cancer (Bultz \& Johansen, 2011). The oncology field is recognizing the need for greater efforts to help patients with cancer cope during treatment and to improve quality of life following treatment. Cancer distress is considered the "sixth vital sign" in cancer care, and clinical practice guidelines for distress screening and management in oncology have been disseminated (National Comprehensive Cancer Network [NCCN], 2010).

\section{The Role of Stress in Cancer Progression}

There is growing evidence that stress responses and adaptation impact tumor growth-promoting processes (increased angiogenesis, migration and invasion, and inflammation) and tumor defense processes (decreased cellular immunity)_all of which can 
impact disease course and survival (Antoni, 2013; Antoni et al., 2006). Andersen, Kiecolt-Glaser, and Glaser (1994) propose that the diagnosis and treatment of cancer increases stress which can then adversely affect not only quality of life but also health behaviors and treatment compliance. Andersen and colleague's biobehavioral model of cancer stress and disease course predicts that stress and health behaviors can directly impact immunity through neuroendocrine and central nervous system innervation, whereas cancer treatment compliance and health behaviors impact the disease processes (local and metastatic). The model also predicts that immunity and disease processes will impact one another and will ultimately impact disease course (Andersen et al., 1994).

Research has found that stress may decrease positive health behaviors (regular exercise, sleep quality, healthful eating) while leading to an increase in negative health behaviors (smoking, alcohol use, compulsive eating; Schuler, Atkinson, \& Philip, 2015). Cancer distress combined with impaired health behaviors is more likely to have an adverse impact on the immune response than either factor alone. Increasing positive behaviors and decreasing negative behaviors may increase immunity while improving quality of life and mood (Schuler et al., 2015).

The enduring somatic threat model (Edmondson, 2014) speculates that cancer-related posttraumatic stress symptoms may be manifested in nonadherence to medical treatment and health recommendationsan avoidance of potent reminders not only of the past traumatic event but also the ongoing somatic threat of cancer (Edmondson, 2014). Women who cope through disengagement-avoiding, ignoring, or withdrawing from stressors or their emotional consequences - have poorer physical health and psychological well-being than those using more engaged coping styles (Kvillemo \& Bränström, 2014). Patients with cancer who endorsed feelings of hopelessness and helplessness experienced $160 \%$ more fatal cancers 6 years later than those that had the lowest level of helplessness (Everson et al., 1996). Those who complete treatment and follow-up have dramatically lower rates of recurrence or death than those who do not (Hershman et al., 2011). Yet the rates of treatment compliance vary widely-from over $90 \%$ (in clinical trials) to $37 \%$ (Li et al., 2000).

\section{Current Psychological Support Interventions in Breast Cancer}

The purpose of psychological support interventions in breast cancer has been to promote awareness and education, provide emotional and social support, assist with problem solving, and improve stress management to increase coping during and after active cancer treatment (Jassim, Whitford, Hickey, \& Carter, 2015). Most of the psychological interventions for breast cancer survivors have not been subjected to randomized controlled trials (RCTs) but those that have combine elements of social support, psychoeducation with a focus on cancer and health-related information, and stress reductions skills, such as progressive muscle relaxation, mindfulness, and guided imagery. Cognitive behavioral therapy (CBT) interventions add problem solving, addressing and learning specific coping skills, and challenging cognitions. Most studies were group interventions. Most did not target traumatic stress symptoms in highly distressed women with breast cancer and have not included evidence-based interventions for posttraumatic stress disorder (PTSD; Jassim, et al, 2015; Nenova et al. 2013).

Psychological interventions have led to mixed results, but some have been found to reduce patient's stress and anxiety and improve moods and quality of life, with some reports of improvements in physical symptoms, such as pain (Galway et al., 2012; Jassim et al., 2015; Mustafa, Carson-Stevens, Gillespie, \& Edwards, 2013; Osborn, Demoncada, \& Feuerstein, 2006). Interventions may be most beneficial for patients with elevated distress (Schneider, Moyer, Knapp-Oliver, Sohl, Cannella, \& Targhetta, 2010). In a review of RCTs of CBT interventions for the treatment of cancer-related traumatic stress symptoms, few studies reported any change in intrusion symptoms, and none reported a positive impact on avoidance symptoms (Nenova et al., 2013).

Do psychological interventions impact disease course and survival? Two meta-analyses of psychosocial interventions found very few well-designed studies (Jassim et al., 2015; Mustafa et al., 2013). In reviews of the impact of psychological intervention on biological outcome variables implicated in tumor progression, psychological interventions have had a positive impact on cancer-specific biological processes, although not all interventions demonstrate positive impacts (Lutgendorf \& Andersen, 2015). There is emerging evidence that interventions that address a woman's most pressing concerns early in diagnosis and treatment may have the greatest impact in improving treatment compliance and reducing stress-related factors that may contribute to biobehavioral processes that effect cancer progression while also decreasing posttraumatic stress symptoms, reducing physical symptoms, and improving quality of life (Antoni, 2013; Chirico, Lucidi, Mallia, D’Aiuto, \& Merluzzi, 2015). 


\section{Eye Movement Desensitization and Reprocessing Therapy}

Eye movement desensitization and reprocessing (EMDR; Shapiro, 2001) is a psychological treatment developed for the treatment of traumatic stress by cancer survivor Francine Shapiro (Shapiro, 1989). Today, 24 RCTs support the use of EMDR therapy for a wide range of trauma populations (Shapiro, 2014). EMDR therapy is recognized as an evidence-based treatment for PTSD and trauma-related disorders and is recommended in clinical practice guidelines of domestic and international organizations (e.g., Bisson \& Andrew, 2007; World Health Organization, 2013). Research has found that individual provision of EMDR (Capezzani et al., 2013) and group applications (Diegelmann \& Isermann, 2003; Jarero et al., 2015) have been effective in the treatment of traumatic stress for patients with cancer.

\section{An Integrative Psychotherapy With an Information Processing Model}

EMDR therapy is guided by Shapiro's (2001) adaptive information processing (AIP) model, which hypothesizes that within each person exists a natural information-processing system that facilitates adaptive learning from life experiences. When blocked, possibly because of high arousal or dissociation, the information-processing system of the brain stores the experience with the emotions, physical sensations, and beliefs that occurred at the time of the event. In the case of a breast cancer diagnosis and treatment, even though medical tests may now show no sign of the disease, the fear, anxiety, thoughts, sensations, and perceptions encoded in an unprocessed memory remain and are the basis of distress that is triggered by current experiences.

In the first two phases (history and preparation) of the eight-phased EMDR therapy, the therapist gathers the client's history and goals, develops a treatment plan that includes the experiences contributing to the current symptoms, and prepares the client for reprocessing. In Phases 3 through 6, the "reprocessing phases" of EMDR therapy, the client focuses on an experience while attending to brief sequential periods of dual-attention bilateral stimulation (BLS) and notices whatever comes into awareness. This leads to a resolution of the maladaptive sensory, emotional, cognitive, and somatic components of the targeted experience. Through these standardized procedures, the contributory memories and present triggers are reprocessed, and positive templates for the future are installed. Phases 7 and 8 focus on stabilization at the end of each session and reevaluation and refinement of the treatment plan.

\section{Resourcing Interventions in EMDR Therapy}

Although the power of EMDR therapy is in the reprocessing of memories that contribute to the client's distress, the incorporation of needed skills for current coping and adaptive learning may be necessary for successful EMDR reprocessing and client stability. Without such positive experiences or counterexamples, there will not be adaptive experience for the targeted memory to link to, and the processing will not progress. This is the focus of EMDR therapy Phase 2, where resource interventions prepare the client for reprocessing.

Calm or safe place imagery, the establishment of a therapeutic relationship, ensuring the ability to be present during processing to facilitate dual awareness, the identification of adequate external supports, and positive activities were the first resources referred to in the early development of EMDR therapy (Shapiro, 2001). Later, resource development and installation (RDI; Leeds, 2009) and other imagery techniques or skills development-often combined with BLS - were added as potential preparation strategies in EMDR therapy (Shapiro, 2007). To date, there are no controlled research studies that have evaluated resource interventions with BLS in EMDR treatment, but adequate Phase 2 preparations, including the ability to shift emotional states, are required for EMDR treatment fidelity (Shapiro, 2001).

When needed, resourcing interventions may enlist imagery, stories, metaphor, somatically focused exercises, Socratic questioning, behavioral experiments, formal instruction, and practice to help patients access existing resources and develop new skills to increase current functioning, capacity for tolerating and regulating strong emotions, and overall sense of self-control (Korn, 2009). Several non-EMDR methods include or emphasize skill building and have demonstrated positive outcomes when subjected to controlled research (Leeds, 2009).

Resources in Peak Performance. Foster and Lendl (1996) cocreated the EMDR peak performance protocol in 1991 to assist individuals in performance anxiety and optimize performance (Foster, 2012). Within the protocol, resourcing procedures are oriented toward peace of mind and empowerment, including generating a client's sense of hope and possibility of realizing their goals of a full and satisfying life (Foster, 2012). Identifying any deficits in requisite skills to achieve success is also crucial, and clients are supported in acquiring the missing skills prior to reprocessing. "If the 
client is worried and feeling distressed about enacting skills that she does not currently possess, attempts to reprocess the anxiety will not be fully successful" (Foster, 2012, p. 214). Guided imagery is used to develop a peaceful place; access and consult an inner coach, advisor, or support team; and accessing and installing past successful experiences and mentally rehearsing future success. Slow, brief sets (6-12) of eye movements are used to strengthen positive associations with resources.

Resource Development and Installation. RDI was first described in 1995 by Leeds (2009) and combines the use of imagery or other interventions to access existing resources or develop needed resources, which are then installed and strengthened with brief sets of BLS (Korn, 2009). The therapist may introduce imagery, sensorimotor, skill-based, or behavioral experiments or experiences as a resource to be installed in RDI (Korn, 2009). In RDI, once a specific challenge has been identified, the client is asked what resource or quality would be helpful in that challenge. Examples may include emotion regulation, containment, grounding, self-soothing, distancing, mindfulness, relationship skills (assertion, boundaries), compassion, strength, courage, and constructive self-talk.

First, a mastery experience, relational experience, or symbolic representation of the needed resource is identified. To access the positive somatosensory experience of the resource, the therapist asks the client to describe the experience or representation in detail. Once the client experiences a vivid and felt sense of the resource, she is instructed to focus on the experience with the positive affect and sensation, while sets of 4-12 eye movements (or bilateral taps or tones) are applied to install the resource. The client is asked to report on the experience, with sets of BLS continuing as long as the feeling is getting stronger or more positive. A cue word is chosen, and strengthened with BLS, and then the client is asked to focus on using the resource with the challenge in the future.

Over time, many such resources may be installed. Ultimately, the patient works on ... future templates, incorporating this new sense of a resourced self into a visualization of effective coping and performance in the future. In addition to resources developed within sessions, any coping successes that the patient reports outside of session (e.g., successful self-soothing, strong, assertive behavior) can be installed using the RDI standard protocol; the patient simply focuses on the mastery experience as the resource target. (Korn, 2009, p. 270)
Other Resources. Summarizing the use of resource interventions in EMDR therapy for complex PTSD, Leeds (2009) lists a wide array of resource-focused interventions including many of the same types used in traditional psychosocial interventions for breast cancer, including relaxation skills, mindfulness and other stress reduction/management skills, exercise and nutrition, assertiveness and communications skills training, and self-care. Additional resources used in psychotherapy listed by Leeds include meditation, prayer, and spiritual practices/beliefs; daily living skills; empowerment skills; creative activities, including art, movement, music, and journaling; and methods for improving self-capacities, including selfregulation skills, biofeedback and neurofeedback, mental rehearsal, and the therapeutic alliance.

\section{Resourcing in Shapiro's EMDR Protocol for IIIness/Somatic Issues}

When first described in 1992, Shapiro's protocol for illness and somatic disorders was influenced by the newly emerging field of psychoneuroimmunology (Shapiro, 2001). The protocol incorporates specific interventions related to illness and health and uses the EMDR standard three-pronged protocol: reprocessing the past memories that have contributed to the disturbance, reprocessing the present situations that continue to be challenging, and developing a future template that includes the incorporation of positive cognitions related to health and well-being. The clinical emphasis is on helping the client to feel empowered and improving her quality of life. Shapiro (2001) takes care to emphasize that EMDR therapy is not intended as a cure or substitution for medical treatment but can support women during any stage of the illness, from diagnosis to survival to recurrence or end of life.

When treating a client with a life-threatening illness such as cancer, Shapiro (2001) stresses the importance of developing a plan to address any fears or problems that require action prior to reprocessing the traumatic events. The resource-focused interventions that she suggests include (a) psychoeducation, (b) problem solving, (c) symptom management techniques, and (d) relaxation training or other coping resources. Shapiro also suggests (e) encouraging clients to access external resources such as supportive group therapy and (f) promoting a plan of self-care that may include the use of complementary health care approaches, such as massage or nutrition. Another intervention involves $(\mathrm{g})$ healing-focused guided imagery to stimulate the immune system and mobilizing resources to promote healing. Compatible with the biobehavioral 
model of cancer stress and disease course, Shapiro's recommended interventions can be expected to increase coping, reduce distress, support treatment adherence, and provide a greater sense of control. The following section describes the provision of each of these types of resources, with brief case examples.

\section{Treatment Planning Considerations}

When planning treatment, "clinicians must determine which problems should be remediated by education, problem solving, or stress management techniques and which are based on dysfunctional information that needs processing" (Shapiro, 2001, p. 104). The EMDR therapy treatment plan should take into account the current phase of her cancer treatment, the demands of cancer treatment and its side effects, her current level of coping, and her goals for therapy. If active cancer treatment has been completed, or if the demands of cancer treatment do not interfere, EMDR therapy can proceed without delay to address the traumatic stress symptoms and distress associated with breast cancer. If intrusive symptoms create significant distress and interfere with action, they should be targeted first-using reprocessing phases when appropriate.

In the first few weeks after diagnosis, women will be focused on managing their emotional reactions without becoming overwhelmed, immobilized, or avoidant; gathering essential information about treatment options and resources for support; and making necessary decisions (Rossman, 2003). Because cancer treatment can be physically demanding and appointment intensive (Holland et al., 2004), EMDR treatment planning should take into account that the client may not be able to attend therapy regularly, may feel fatigued or ill, or may have unexpected, pressing needs that preempt plans for reprocessing. EMDR reprocessing may need to be delayed if she has major difficulties with emotional regulation, life management, or cannot attend counseling often enough. Resource-focused interventions, which do not require regular appointments, may be particularly valuable during active breast cancer treatment.

\section{Identify Realistic Fears}

Shapiro (2001) recommends first identifying realistic fears that must be addressed prior to reprocessing memories. Cancer-specific measures of distress can reveal realistic fears and problems requiring immediate attention. The easily administered distress thermometer and accompanying problem list (NCCN, 2010) provide quick identification of distress stemming from emotional, spiritual, or religious concerns; practical or family issues; or physical problems. The Impact of Cancer Version 2 (Crespi, Ganz, Petersen, Castillo, \& Caan, 2008) can be used following completion of active cancer treatment. Both instruments are available in the public domain. Given the ever-changing challenges that are inherent in cancer treatment, these measures should be administered regularly.

\section{Using EMDR Resource Strategies in Breast Cancer}

EMDR resourcing methods typically involve asking the patient to generate and then focus on a positive experience, skill, or capacity while engaging in sets of BLS (generally fewer and slower) to allow a deepening of the positive associations. Resourcing can be used for stabilization, ego-strengthening, or skill-based approaches. For example, once a woman is able to learn and have a positive experience (mental or in-vivo) of effectively asking for help in a social or medical environment, the clinician can invite the client to focus on the experience-by accessing its positive sensory, affective, and the mental qualities — and add a few sets (four to eight) of BLS. This is thought to enhance the skills and encourage further generalization of the resource. In future rehearsal, the client imagines using the resource, skill, or capacity while encountering an anticipated challenge-leading to a greater sense of mastery and confidence in the resource.

\section{Considerations When Using Guided Imagery in Resourcing}

In addition to its use in developing skills and increasing internal capacities, guided imagery can be used to help teach relaxation, to relieve physical symptoms, to stimulate healing responses in the body, and to increase tolerance of cancer treatments (Rossman, 2003). For maximum effectiveness in guided imagery, the therapist should use language that includes not only visual images but also sounds, smells, sensations, dialogue, and emotional content by inviting a client to allow whatever she sees, feels, hears, tastes, senses, or experiences to evolve naturally in her imagination. The imagery should be interactive and dynamic rather than rigidly scripted. By inviting imagery that is evoked from the client rather than guiding the client in a predetermined experience, imagery accesses the client's own inner resources to meet the identified challenge. The client can be invited to notice what comes into awareness when a particular imagery exercise is used and explore what emerges with curiosity. This form of imagery is inherently client-centered 
and empowering. With the possibilities limited only by her imagination, the client can make adjustments to the imagery to best suit her needs.

Case Example: Interactive Safe Place Imagery for Coping. Jamie learned about guided imagery years before being diagnosed with cancer. She described her evolving safe place that offered respite not only from her fears but also from the physical effects of cancer treatments:

Early after my diagnosis, I used my safe place quite literally as a place for safety. I retreated to my sunny little place on the edge of a forest in search of brief periods of relief from fear, pain, nausea, and exhausting crying jags. As pathetic as that sounds, I was very grateful for any little bit of distance those retreats put between me and my most unwelcome predicament.

Any difficulty I had ever faced in the past, and there were some big ones, held the promise that time heals all wounds. This was different. There were no promises. No one said, "You're going to be okay, we're going to cure you." No one even said, "We caught it early ..." In fact, there was a very real possibility that time would reveal worse to come. There was no rest, no place of safety. So I did the best I could to make my own.

I wrapped myself in a soft, pink blanket and curled up in a nest-like platform formed at the low junction of three giant oak trees. I buried my head and prayed that everything would be okay. To whom I was praying I did not know. It never really mattered to me before. But now that my life was aggressively threatened, suddenly it mattered....

When anemia caused by chemotherapy chilled me to the bone, I installed heat in the trunk of the tree and turned it up full blast with an irreverent lack of fear of the consequences of heating the whole outdoors. I added AC to combat the heat waves of my hot flashes. When my body ached and I bruised from nothing more than kneeling or pressing against a hard surface, I lined my nest with sheep's wool. And when nightmares kept me awake, I made a campfire to ward off all evil while I slept. (Peck, 2006)

\section{Resourcing Through Psychoeducation}

Providing psychoeducation may be helpful when distress is caused by being overwhelmed or confused about cancer information, treatment options, risks, prognosis, and how to process the information with loved ones (Galway et al., 2012). Whereas an oncology specialist will provide the information about her specific cancer, tumor characteristics, and oncology treatment options, a therapist can help a woman integrate the information. Psychoeducational interventions that prepare women for consultation appointments with their doctors-including identifying questions and concerns, generating a printed agenda, and skills training to improve communication with their doctors - can be helpful (Sepucha, Belkora, Mutchnick, \& Esserman, 2002).

Case Example of Psychoeducation for Medical Appointments. Mary had just been diagnosed with breast cancer and wanted help to "keep it together" to understand the complicated medical information she was receiving. I gave Mary the Livestrong Guidebook, Planner and Journal (Armstrong, 2009)-a two-volume set that helps survivors learn about their cancer and treatments; find medical care, resources, and support services; learn how to prepare for appointments and what to ask doctors; make life and health decisions; learn about financial and insurance issues; and keep track of their cancer journeys. Mary found it a helpful companion and reference.

Psychoeducation may also include helping the client understand the interplay between cancer, stress, and behaviors, and how stress management, coping skills, social supports, problem solving, cancer treatment adherence, exercise, nutrition, and improved health habits can positively impact not only her quality of life, mood, and fears of recurrence but also her overall health and disease course. I find the book Anticancer: A New Way of Life by Dr. David Servan-Schreiber (2009) an inspiring and informative resource that provides useful information consistent with biobehavioral goals, including EMDR therapy to address feelings of helplessness and hopelessness that may contribute to the biological processes involved in tumor growth.

Case Example of Psychoeducation to Dispel Concerns About Her "Attitude." Ann heard that she needed to be "positive" to survive cancer. She lamented, "If I have to be positive, then I'm a dead woman. I've always been told I had a bad attitude." From earlier work, I knew Ann to be bright and outspoken—she was not inclined toward passive compliance. I told her that some research found that women who are actively engaged in treatment decisions and assertively communicate with providers are more likely to have better outcomes than those who are passively positive, in denial, or feel helpless or hopeless. Ann felt relieved by this information. 


\section{Resourcing Through Problem Solving and} Skill Development

Of course, not all challenges requiring immediate attention can be resolved through psychoeducation. Patients may feel overwhelmed with the demands of juggling medical decisions, treatment, work, financial, and family concerns while feeling physically, emotionally, and mentally depleted. For some, dissociation may interfere with the ability to integrate information or take action. Minor problems can feel insurmountable, and she may lose her sense of control and efficacy because her life decisions and plans are steered by others.

The clinician can assist the patient in articulating her challenges and facilitate problem solving to create an action plan to address real needs and fears. If deficient, skill training and other resourcing may be required for the client to carry out the plan. For example, some women will benefit from resourcing interventions to increase presence and manage dissociation. For others, communication skills training may help to carry out a plan to ask a friend or family member to attend appointments with them, or to ask for practical help (transportation, childcare) that will allow them to attend medical appointments, or to assist them in expressing their concerns or needs with their medical providers. Assertive communication may be taught in session, including role-playing. These new skills can then be strengthened through imagery, including future rehearsal with an upcoming challenge. Positive internal states derived from the rehearsal and imagined or actual use of a skill can be installed and strengthened with BLS.

Case Example of Problem Solving for Isolation and Resourcing for Confidence. Not only was Mary intimidated by doctors, she also felt alone. We began to problem solve both her feelings of isolation and intimidation. I let her know that many women find it helpful to have a support person attend oncology appointments to provide practical and emotional support, take notes, and provide an extra set of ears or voice during times of heightened anxiety or vulnerability. We created a diagram of her social network and identified who she could ask for help.

In RDI, Mary identified "confidence" as the resource that would allow her to ask for help from others and feel less intimidated by doctors. After identifying and exploring an experience of confidence when talking with her daughter's teachers, I asked her to step into the experience fully and added BLS to install and strengthen the internal state. Using future rehearsal, I invited Mary to imagine meeting with her doctor and asking questions with confidence. She felt buoyed by her plan. Adding another set of BLS, she added the statement "I can ask for what I need."

\section{Resourcing Through a Plan of Daily Self-Care}

Women can develop a plan for daily self-care (Shapiro, 2014) or an "anticancer" regimen (Servan-Schreiber, 2009) that includes attention to physical, emotional, social, and spiritual needs. The plan should be dynamic, evolving, and realistic. The therapist can help the patient to identify, plan, and engage in positive health behaviors (exercise, nutrition, sleep hygiene); identify, plan, and reduce negative health behaviors (smoking, compulsive eating, substance use); and develop and strengthen mental and emotional states and behaviors that positively impact biological processes involved in cancer progression and cancer immunity.

While regularly monitoring and updating her plan, EMDR resourcing or reprocessing can help to ensure success. The plan should be reviewed regularlyparticularly as cancer treatment ends. With the loss of the structure of active cancer treatment and loss of contact with caregivers and other patients, anxiety and fears of recurrence may increase (Stanton, Rowland, \& Ganz, 2015). A plan of self-care for the next chapter of her life can help quell anxieties and increase confidence.

Case Example of Adding Self-Care to Her Anticancer Plan and Inner Healer Imagery. Gail's attention to her physical health was nearly impeccable. When reading Anticancer: A New Way of Life (Servan-Schreiber, 2009), she recognized that her tendency to put other's needs first led to feelings of resentment, powerlessness, and despair; mental states that Servan-Schreiber (2009) warned could negatively impact immunity. As part of her anticancer plan, Gail committed to 8 hours of sleep, taking charge of meeting her own needs and not deferring to the need of others.

To problem solve anticipated challenges, she agreed to use imagery to invite an inner advisor/healer for help, an empowering technique to help a woman find answers from her own unconscious. A powerful but kindly mother bear appeared in the imagery. As Gail became more comfortable with the inner dialogue, she was touched by the bear's fierce protectiveness of her needs, and it's no nonsense solutions. Slow sets of BLS strengthened the clear plan for self-care.

\section{Resourcing Through Symptom Management}

Physical symptoms associated with active cancer treatment have been responsive to psychological interventions, with improvements in sleep, fatigue, pain, and nausea. For example, the light stream exercise taught 
in most EMDR training sessions can be readily adapted for pain management (Shapiro, 2001). Guided imagery can be used in session to manage pain, nausea, fatigue, or insomnia, and enhanced with BLS. The client should be encouraged to use the imagery between sessions. An example is Jamie's use of safe place imagery to manage physical symptoms from cancer treatment.

With every client who is diagnosed with cancer, I encourage the use of cancer-specific guided imagery recordings at home (Kwekkeboom et al., 2012; Rossman, 2003). Even when she does not feel well enough to attend appointments, daily use of imagery recordings provides help to increase relaxation and mindfulness, cope with treatment, manage fears, and envision healing. In a later section of this article, I describe a client who used Simon-type imagery and recordings to reduce chemotherapy-induced nausea.

\section{Resourcing Through External Resources}

External Resources for Coping and Distress Management. Resources that support coping and distress management may be found in formal and informal social and emotional supports as well as programs, activities, or other sources, including websites, books, or videos. Some examples include (a) local and online breast cancer support groups; (b) social media groups such as CaringBridge (https://www .caringbridge.org/); (c) psycho-oncology consultants or patient navigators to facilitate access to resources; (d) classes or groups such as mindfulness, yoga, taichi, and art or dance workshops; and (e) other community wellness resources.

Case Example of External Resource to Increase Social Support Combined With Resource Development and Installation. Because Mary struggled with fears of isolation, I encouraged her to explore CaringBridge, which provides a free personal, protected webpage to post health updates, receive words of encouragement from friends and family, and coordinate care such as bringing a meal or caring for pets. At her next session, Mary was touched by the messages of support and offers of practical help she was receiving. It was clear that the support she was experiencing could serve as a vital resource.

Using RDI, I invited Mary to step into the experience while noticing the accompanying imagery thoughts, emotions, and sensations. Brief, slow sets of BLS were applied as I invited her to savor the experience. She chose the statement, "I am loved and supported even when alone," and "accepting love heals me," which were strengthened with BLS. In future rehearsal, Mary imagined her husband being away for work. With the internal resource of connection and support, she could anticipate enjoying solitude and taking initiative socially. The external resource of CaringBridge paved the way for a long desired internal resource.

External Resources for Health and Treatment Adherence. External resources that support health and treatment adherence may include referral to (a) reliable sources of information about breast cancer, treatment, and survivorship; (b) traditional and complementary medicine practitioners, such as massage and acupuncture; (c) community resources to address barriers to access to medical care, such as a patient navigator; and (d) community resources to support engagement in treatment, manage physical symptoms, and support health behaviors, such as walking groups, exercise programs, nutrition classes, or 12 step support groups.

Case Example of External Resources to Support Health. Ann heard that the timing of surgery and her hormone cycle could impact treatment outcomes. When her oncologist dismissed her concern, she felt confused and fearful. I referred her to a regional cancer center for a second opinion, and a naturopath for complementary and alternative treatment approaches. With the added input, Ann was able to feel more comfortable with the timing of her surgery. Later, these same resources were helpful when she was faced with other difficult choices in treatment.

The Therapeutic Relationship as a Resource. The therapeutic relationship is an essential resource acting as a container and safe place where the client's greatest fears and hopes can be held without judgment. A therapist must have adequate affect tolerance to be able to provide a safe container for the client and avoid inadvertently responding with the types of platitudes, false hopes (or dire predictions), or judgments that may come from others. Just as our client must live with uncertainty, we must also accept that we do not know what the outcome will be.

\section{Resourcing Through Healing-Focused Imagery}

Guided imagery to anticipate, prepare, and support treatment sessions can increase physical and emotional tolerance of cancer treatment. Imagining surgery, radiation treatment, and chemotherapy sessions being effective can help identify concerns and develop helpful resources. As mentioned earlier, home use of audio recordings can maximize these benefits (Rossman, 2003). Some cancer-specific recordings include imagery to consult with an "inner healer" for decision making, symptom management, and to support 
health behaviors. This type of imagery can also be helpful during times of worry or fears of the future.

Case Example of Wisdom Figure for Hope and Healing. Jamie wrote of her experience of a wisdom figure that evolved in imagery:

An old Indian woman (a crone, I'd say) with long, salt-and-pepper braids came to me on the slope of a hill overlooking the generic Alps. The wrinkles on her leathered face were hard-earned evidence of worldly experience and gave the impression of wisdom. I sensed that she held a position of importance, maybe as a medicine woman or a shaman. She surveyed the view and gestured as if she were offering me the entire world-her world. Together we observed the direct point of the EMDR session. When I telepathically indicated I understood the message, she came toward me and hugged me like a proud and loving parent. As we hugged, we melted into each other.

... [I] burst into tears with the profound realization that this beautiful, wise, old woman was a part of me and I would always have her with me. And since then, I have never been completely alone. She is often there in my safe place. Always working on a hand-craft project, she sits quietly on the stone slabs that were once intended to be my alter. I still don't know who she is or to whom she reports in the big scheme of things, but she is the one I deliver my prayers to. She is the one who shares the burden of my fear and anxiety without judgment. She is the only one who has ever told me "everything is going to be okay. (Peck, 2006)

Simonton-Type Imagery. Shapiro (2001) recommends Simonton-type imagery which involves visualizing cancer cells being weakened, neutralized and/ or destroyed and leaving the body, while the immune system gains increasing strength and vigor. Shapiro cautions that "self-healing exercises" should be explored only after addressing any self-blame for her cancer. Bolstering her healing processes does not make a woman responsible for her disease or her physical recovery. It is thought that this type of imagery may increase the patient's confidence and comfort in treatment, improve her quality of life, manage stress and anxiety, adhere to cancer treatment, and potentially stimulate the body's natural cancer-fighting properties and physical recovery. It may help to reduce worry and feelings of helplessness or powerlessness.

Case Example of Simonton-Type Imagery That Reduces Nausea Symptoms. Gail began therapy in hopes of managing the nausea and anxiety she experienced before and during chemotherapy. She could not help but think of the chemotherapy as poison. After reading suggested information about cancer treatment, we talked about how chemotherapy destroys cancer cells and how her immune system functions to support healthy cells and inhibit cancer.

Through interactive imagery, Gail developed dynamic, healing imagery that included (a) a deepening state of ease and relaxation as she explored a personal healing place, (b) chemotherapy effectively destroying or neutralizing cancer cells, (c) her immune system clearing the way to full health, and (d) experiencing herself in the not too distant future with restored health and engagement in life. Once she was readily able to imagine this, I asked her what positive belief accompanied the imagery. Gail said, "My body welcomes help, knows how to heal, and embraces life." I invited her to hold the dynamic, healing imagery with the positive belief while I added BLS to strengthen and install the resource.

Gail used the imagery at home with the positive belief at least once a day and frequently during chemotherapy. She listened to an audio recording that supported this imagery. Gail was able to feel more confident and relaxed before and during chemotherapy and no longer experienced anticipatory nausea. With the chemotherapy-induced nausea and vomiting significantly reduced, Gail bounced back more quickly from the treatment. She found hope and strength in the imagery and used it whenever she experienced any fears or worries about her future health.

\section{Resourcing With Relaxation and Mindfulness}

Patients with cancer may struggle with physical stress responses and arousal which may contribute to anxiety, depression, and increased physical symptoms from treatment. As mentioned previously in this article, the effects of stress can be deleterious to biological processes impacting health and health behaviors (Schuler et al., 2015). Interventions aimed at reducing arousal and increasing relaxation may improve physical and psychological outcomes. Some strategies involve working directly with the body to decrease arousal. These include breathing techniques such as diaphragmatic breathing and square breathing, yoga and tai-chi practices, walking or other exercise, progressive muscle relaxation, and heart rate coherence biofeedback (Servan-Schreiber, 2009). Mindfulness, imagery practices such as calm/ safe place imagery or imagining her body comfortably relaxed, and interventions to manage worry may also decrease stress and increase quality of life. 
Case Examples of Client-Centered Relaxation Practice. I invite women to choose daily relaxation or stress management method(s) to suit their needs and lifestyle. Gail chose walking and yoga. After practicing in session, Mary used a portable biofeedback device. Each day, Ann visited her healing place imagery, consulted with her inner support team, and listened to music.

Music may provide relief from symptoms and improve mood by facilitating a shift to a preferred (powerful, relaxed, strong, resilient) state. Many survivors compile a personal playlist for specific situations such as times of worry, chemo treatments, or while exercising to reduce anxiety or physical discomfort. Through music, women can connect to times of joy, resilience, power, or ease - a potent reminder that this is only a chapter of her life journey.

Mindfulness. Women with breast cancer may benefit from mindfulness practice (Bower et al., 2015) which is "paying attention in a particular way: on purpose, in the present moment, and nonjudgmentally" (Kabat-Zinn, 1994, p. 4). Mindfulness practice can teach women to identify and tolerate distressing or painful thoughts, feelings, and sensations. In addition to a reduction in distress, a woman may gain a sense of mastery rather than feeling helpless to her experiences.

Managing Worry. Long after pronounced "cancerfree," fears of recurrence, cancer-related intrusions, and worry may persist (Stanton et al., 2015). Although the statistics and probabilities reported by her doctor represent population averages, they do not determine what an individual patient's response will be, cannot foretell the best course of action, or whether there will be a recurrence of cancer. Consequently, some women are immobilized by worry and fear-which can be triggered by unexplained sensations, medical appointments, news reports, or simply seeing her mastectomy scar. Resourcing intervention can help women to manage worry, support decision making, and increase quality of life and engagement in treatment.

Case Example: Resource Development and Installation to Discriminate Worry From Intuition (Murray, 2010). After 2 years of doctors dismissing her concerns that she had cancer, Jamie was diagnosed at age 41 with Stage 3 breast cancer. Although in remission, she was hypervigilant to any unexplained pain or symptom. EMDR reprocessing took away the free fall panic and fear of recurrence, but she wanted to feel confident in her ability to know what deserved concern and what did not. We used RDI to explore past experiences of unhelpful worry and other times when she felt an accurate, strong intuition that something was wrong. As she became familiar with each, BLS was added to strengthen her confidence in being able to tell them apart. Jamie described the difference: "Worry involves a lot of talking inside my head and tends to be about the future. Intuition comes from my gut and guides me only with issues at hand" (Peck, 2006). By trusting her intuition, Jamie felt a greater sense of calm and confidence in her ability to take care of herself.

\section{Discussion}

Consistent with biobehavioral goals, EMDR resourcing for women with breast cancer may support coping, quality of life, health behaviors, treatment compliance, and the body's natural anticancer processes. This article offers a few examples of the wide array of resource-focused interventions an EMDR therapist might use during active cancer treatment. Some of the same resources may also serve to prepare a woman for the reprocessing phases of EMDR therapy, and many may be beneficial long after cancer treatment is past.

In my work with the women described in this article, Shapiro's (2001) EMDR protocol for illness and somatic disorders and the AIP model guided the use of EMDR resourcing. During active cancer treatment, therapy addressed the realistic fears and challenges women faced. The resourcing interventions served as a counterbalance to the demands of cancer-related experiences. However, the greatest relief came from EMDR therapy's comprehensive processing of cancer-related experiences, triggers, future fears, and installation of positive future templates. It cannot be overemphasized that resourcing interventions should not unnecessarily delay the reprocessing phases of EMDR therapy and should be tailored to a client's specific needs. There is no one size fits all in the pacing of EMDR therapy.

Hippocrates believed that the body contains an innate power to rebalance and heal itself. To treat injury or illness, his treatments were focused on simply easing this natural self-healing process. His therapeutic approach was based on "the healing power of nature" (vis medicatrix naturae in Latin). Essential to processes of rebalancing and self-healing are the growth of new cells and death of old cells: a process that is clearly out of balance within the immune system during the development and progression of breast cancer. Both the body and mind play a role in this balance, as stress responses and coping can impact tumor growth and tumor defense processes. 
Foundational to EMDR therapy, Shapiro (2007) has proposed that within each person is an information processing system that serves to efficiently clear the stored residue from psychological wounds while forging connections to adaptive experiences. When unobstructed, the AIP system restores and maintains balance and psychological health by integrating learning within memory networks. It also requires balance-in the form of dual awareness and accessible, adaptive information to counterbalance the difficult experiences of life. When out of balance, the disturbing affects, sensations, sensory information and beliefs are stored as they were at the time of the original experience-unable to be assimilated into larger adaptive memory networks.

EMDR therapy's eight phases and three-pronged approach strive to restore balance in information processing and support health, growth, and resilience. While the greatest power of EMDR lies in the efficient reprocessing of dysfunctionally stored experiences that contribute to current distress and symptoms, the AIP system cannot function properly without sufficient balancing effects of dual awareness and accessible adaptive experiences. Just as a physician must monitor a patient's physical functioning and equilibrium during oncology treatment to assure no harm is done, so too, must EMDR therapists assure adequate stability and access to adaptive information and resources in preparation for and during EMDR reprocessing. When the demands of cancer treatment, irregular attendance in counseling, or insufficient client stability require a delay in the reprocessing phases of EMDR therapy, EMDR resourcing interventions are the right "medicine" to give. However, research is needed to evaluate the impact of EMDR therapy resourcing interventions and EMDR standard protocol reprocessing on women's distress, coping, treatment adherence, health behaviors, post traumatic stress symptoms, fears of recurrence, health outcomes and quality of life during and following active treatment for breast cancer.

\section{References}

Andersen, B. L., Kiecolt-Glaser, J. K., \& Glaser, R. (1994). A biobehavioral model of cancer stress and disease course. American Psychologist, 49(5), 389-404.

Antoni, M. H. (2013). Psychosocial intervention effects on adaptation, disease course and biobehavioral processes in cancer. Brain, Behavior, and Immunity, 30(Suppl.), S88-S98.

Antoni, M. H., Lutgendorf, S., Cole, S., Dhabhar, F., Sephton, S., McDonald, P., . . Sood, A. (2006). The influence of bio-behavioural factors on tumor biology: Pathways and mechanisms. Nature Reviews: Cancer, 6, 240-248.
Armstrong, L. (2009). Livestrong guidebook, planner and journal. Austin, TX: Armstrong Foundation.

Bisson, J., \& Andrew, M. (2007). Psychological treatment of post-traumatic stress disorder (PTSD). Cochrane Database of Systematic Reviews, (3), CD003388.

Bower, J. E., Crosswell, A. D., Stanton, A. L., Crespi, C. M., Winston, D., Arevalo, J., . . Ganz, P. A. (2015). Mindfulness meditation for younger breast cancer survivors: A randomized controlled trial. Cancer, 121(8), 1231-1240.

Bultz, B. D., \& Johansen, C. (2011). Screening for distress, the 6th vital sign: Where are we, and where are we going? Psycho-Oncology, 20, 569-571.

Capezzani, L., Ostacoli, L., Cavallo, M., Carletto, S., Fernandez, I., Solomon, R., . . . Cantelmi, T. (2013). EMDR and CBT for cancer patients: Comparative study of effects on PTSD, anxiety, and depression. Journal of EMDR Practice and Research, 7(3), 134-143.

Chirico, A., Lucidi, F., Mallia, L., D’Aiuto, M., \& Merluzzi, T. V. (2015). Indicators of distress in newly diagnosed breast cancer patients. PeerJ, (3), e1107.

Chopra, I., \& Kamal, K. M. (2012). A systematic review of quality of life instruments in long-term breast cancer survivors. Health and Quality of Life Outcomes, 10, 14.

Crespi, C. M., Ganz, P. A., Petersen, L., Castillo, A., \& Caan, B. (2008). Refinement and psychometric evaluation of the impact of cancer scale. Journal of the National Cancer Institute, 100, 1530-1541.

Diegelmann, C., \& Isermann, M. (2003, May). Trauma exposure and crisis intervention with CIPBS. Poster presented at the VIII European Conference on Traumatic Stress. Berlin, Germany.

Edmondson, D. (2014). An enduring somatic threat model of posttraumatic stress disorder due to acute life-threatening medical events. Social and Personality Psychology Compass, 8(3), 118-134.

Everson, S. A., Goldberg, D. E., Kaplan, G. A., Cohen, R. D., Pukkala, E., Tuomilehto, J., \& Salonen, J. T. (1996). Hopelessness and risk of mortality and incidence of myocardial infarction and cancer. Psychosomatic Medicine, 58(2), 113-121.

Foster, S. L. (2012). Integrating positive psychology applications into the EMDR peak performance protocol. European Review of Applied Psychology, 62(4), 213-217.

Foster, S., \& Lendl, J. (1996). Eye movement desensitization and reprocessing: Four case studies of a new tool for executive coaching and restoring employee performance after setbacks. Consulting Psychology Journal, 48(3), $155-161$.

Galway, K., Black, A., Cantwell, M., Cardwell, C. R., Mills, M., \& Donnelly, M. (2012). Psychosocial interventions to improve quality of life and emotional wellbeing for recently diagnosed cancer patients. Cochrane Database of Systematic Reviews, (11).

Hershman, D. L., Shao, T., Kushi, L. H., Buono, D., Tsai, W. Y., Fehrenbacher, L., . . Neugut, A. I. (2011). Early discontinuation and non-adherence to adjuvant hormonal therapy are associated with increased mortality 
in women with breast cancer. Breast Cancer Research and Treatment, 126(2), 529-537.

Holland, J., Herdman, R., \& Hewitt, M. (Eds.). (2004). Meeting psychosocial needs of women with breast cancer. Washington, DC: National Academies Press.

Jarero, I., Artigas, L., Uribe, S., García, L. E., Cavazos, M. A., \& Givaudan, M. (2015). Pilot research study on the provision of the eye movement desensitization and reprocessing integrative group treatment protocol with female cancer patients. Journal of EMDR Practice and Research, 9(2), 98-105.

Jassim, G. A., Whitford, D. L., Hickey, A., \& Carter, B. (2015). Psychological interventions for women with non-metastatic breast cancer. Cochrane Database of Systematic Reviews, (5), CD008729.

Kabat-Zinn, J. (1994). Wherever you go there you are: Mindfulness meditation in everyday life. New York, NY: Hyperion Books.

Korn, D. L. (2009). EMDR and the treatment of complex PTSD: A review. Journal of EMDR Practice and Research, 3(4), 264-278.

Kvillemo, P., \& Bränström, R. (2014). Coping with breast cancer: A meta-analysis. PLoS One, 9(11), e112733.

Kwekkeboom, K. L., Abbott-Anderson, K., Cherwin, C., Roiland, R., Serlin, R. C., \& Ward, S. E. (2012). Pilot randomized controlled trial of a patient-controlled cognitive-behavioral intervention for the pain, fatigue, and sleep disturbance symptom cluster in cancer. Journal of Pain and Symptom Management, 44(6), 810-822.

Laubmeier, K. K., \& Zakowski, S. G. (2004). The role of objective versus perceived life threat in the psychological adjustment to cancer. Psychology \& Health, 19(4), 425-437.

Leeds, A. M. (2009). Resources in EMDR and other traumafocused psychotherapy: A review. Journal of EMDR Practice and Research, 3(3), 152-160.

Li, B. D., Brown, W. A., Ampil, F. L., Burton, G. V., Yu, H., \& McDonald, J. C. (2000). Patient compliance is critical for equivalent clinical outcomes for breast cancer treated by breast-conservation therapy. Annals of Surgery, 231(6), 883-889.

Lutgendorf, S. K., \& Andersen, B. L. (2015). Biobehavioral approaches to cancer progression and survival: Mechanisms and interventions. American Psychologist, 70(2), 186-197.

Matsuoka, Y., Nagamine, M., \& Uchitomi, Y. (2006). Intrusion in women with breast cancer. In N. Kato, M. Kawata, \& R. K. Pitman (Eds.), PTSD: Brain mechanisms and clinical implications (pp. 169-178). Tokyo, Japan: Springer Publishing.

Murray, K. (2010, September/October). EMDR to reduce fears of recurrence in breast cancer. Paper presented at the 15th EMDR International Association Conference, Minneapolis, MN.

Mustafa, M., Carson-Stevens, A., Gillespie, D., \& Edwards, A. G. (2013). Psychological interventions for women with metastatic breast cancer. Cochrane Database of Systematic Reviews, (6), CD004253.

National Comprehensive Cancer Network. (2010). NCCN clinical practice guidelines in oncology: Distress management. Version 3.2015. Fort Washington, PA: Author.

Nenova, M., Morris, L., Paul, L., Li, Y. P., Applebaum, A. P., \& DuHamel, K. P. (2013). Psychosocial interventions with cognitive-behavioral components for the treatment of cancer-related traumatic stress symptoms: A review of randomized controlled trials. Journal of Cognitive Psychotherapy, 27(3), 258-284.

Osborn, R. L., Demoncada, A. C., \& Feuerstein, M. (2006). Psychosocial interventions for depression, anxiety, and quality of life in cancer survivors: Meta-analyses. International Journal of Psychiatry in Medicine, 36, 13-34.

Peck, J. M. (2006). A safe place. Unpublished manuscript.

Pérez, S., Galdón, M. J., Andreu, Y., Ibáñez, E., Durá, E., Conchado, A., \& Cardeña, E. (2014). Posttraumatic stress symptoms in breast cancer patients: Temporal evolution, predictors, and mediation. Journal of Traumatic Stress, 27(2), 224-231.

Phelps, E. A., \& Sharot, T. (2008). How (and why) emotion enhances the subjective sense of recollection. Current Directions in Psychological Science, 17, 147-152.

Rossman, M. L. (2003). Fighting cancer from within: How to use the power of your mind for healing. New York, NY: Henry Holt.

Schneider, S., Moyer, A., Knapp-Oliver, S., Sohl, S., Cannella, D., \& Targhetta, V. (2010). Pre-intervention distress moderates the efficacy of psychosocial treatment for cancer patients: A meta-analysis. Journal of Behavioral Medicine, 33(1), 1-14.

Schuler, T. A., Atkinson, T. M., \& Philip, E. J. (2015). Cancer. In F. Andrasik, J. L. Goodie, \& A. L. Peterson (Eds.), Biopsychosocial assessment in clinical health psychology (pp. 195-213). New York, NY: Guilford Press.

Sepucha, K. R., Belkora, J. K., Mutchnick, S., \& Esserman, L. J. (2002). Consultation planning to help breast cancer patients prepare for medical consultations: Effect on communication and satisfaction for patients and physicians. Journal of Clinical Oncology, 20(11), 2695-2700.

Servan-Schreiber, D. (2009). Anticancer: A new way of life. New York, NY: Viking.

Shapiro, F. (1989). Efficacy of the eye movement desensitization procedure in the treatment of traumatic memories. Journal of Traumatic Stress Studies, 2, 199-223.

Shapiro, F. (1992, May). Stray thoughts. EMDR Network Newsletter, 2(1), 1-2.

Shapiro, F. (2001). Eye movement desensitization and reprocessing: Basic principles, protocols, and procedures (2nd ed.). New York, NY: Guilford Press.

Shapiro, F. (2007). EMDR, adaptive information processing, and case conceptualization. Journal of EMDR Practice and Research, 1(2), 68-87.

Shapiro, F. (2014). The role of eye movement desensitization and reprocessing (EMDR) therapy in medicine: Addressing 
the psychological and physical symptoms stemming from adverse life experiences. The Permanente Journal, 18(1), 71-77.

Stanton, A. L., Rowland, J. H., \& Ganz, P. A. (2015). Life after diagnosis and treatment of cancer in adulthood: Contributions from psychosocial oncology research. American Psychologist, 70(2), 159-174.
World Health Organization. (2013). Guidelines for the management of conditions specifically related to stress. Geneva, Switzerland: Author.

Correspondence regarding this article should be directed to Katy Murray, 3627 Ensign Road NE, Ste B., Olympia, WA 98506. E-mail: katymurraymsw@gmail.com 